\title{
Time Course of Elevations in Plasma Olprinone Concentration during Pediatric Cardiac Surgery
}

\author{
Satoshi Kurokawa, Minoru Nomura \\ Department of Anesthesiology, Faculty of Medicine, Tokyo Women's Medical University, Tokyo, Japan. \\ Email: satokuro@sea.plala.or.jp
}

Received January $10^{\text {th }}, 2013$; revised February $15^{\text {th }}, 2013$; accepted March $2^{\text {nd }}, 2013$

\begin{abstract}
Purpose: Little research has been reported to date on the usefulness of olprinone in pediatric cardiac surgery, and no standard pediatric infusion protocol is currently established. Our study sought to confirm that the regimen described herein rapidly achieves the requisite plasma olprinone concentrations. Methods: For the purposes of our study, we enrolled 13 patients: 7 biventricular repair candidates and 6 Fontan-type operation candidates. We administered a continuous infusion of olprinone to our study subjects at $0.3 \mu \mathrm{g} / \mathrm{kg} / \mathrm{min}$ with no loading dose starting approximately 30 minutes ( $\mathrm{min}$ ) before weaning from cardiopulmonary bypass (CPB). We performed blood sampling at 15, 30, 45, 60, 90, and $120 \mathrm{~min}$ after the start of infusion and at the same elapsed intervals after separation from CPB. We measured plasma olprinone concentrations using ultra-fast liquid chromatography. Results: We observed effective plasma olprinone concentrations $(>20 \mathrm{ng} / \mathrm{ml}$ ) at $30 \mathrm{~min}$ after weaning from $\mathrm{CPB}$, or at $60 \mathrm{~min}$ after the start of infusion. Conclusion: We conclude that continuous olprinone infusion at $0.3 \mu \mathrm{g} / \mathrm{kg} / \mathrm{min}$ without a loading dose initiated immediately after the release of aortic cross-clamping or immediately after the completion of all surgical procedures quickly and reliably achieves effective plasma concentrations.
\end{abstract}

Keywords: Olprinone; Phosphodiesterase-3 Inhibitor; Plasma Concentration; Pediatric Cardiac Surgery; Weaning from Cardio-Pulmonary Bypass

\section{Introduction}

The effects of milrinone on hemodynamics and outcomes in pediatric cardiac surgery have been extensively investigated. A large multi-center study in North America (the PRIMACORP study) demonstrated high-dose milrinone significantly reduced mortality or the incidence of low cardiac output syndrome (LOS) after pediatric cardiac surgery and that even low-dose milrinone likewise tended to reduce mortality or LOS development, although low-dose results failed to reach statistically significant levels [1]. Milrinone is now widely administered to patients with congenital heart disease.

Olprinone is a phosphodiesterase-3 (PDE-3) inhibitor developed and commercially available in Japan since 1996. Several studies involving adults show olprinone reduces SVR and increases CI significantly during or after cardiac surgery, including coronary artery bypass graft or valve surgery [2-5]. However, the manner of administration has differed from study to study, and no standard protocol has been established. In contrast to milrinone, little data on olprinone is available at this time, data on use during pediatric cardiac surgery in particular. Even optimal regimens for achieving effective blood concentrations remain uncertain. Our study sought to confirm that our infusion method rapidly achieves effective blood concentrations on weaning from CPB during pediatric cardiac surgery.

\section{Methods}

\subsection{Materials}

After local ethics committee approval and informed parental consent, 13 pediatric patients enrolled in this study underwent cardiac surgery under $\mathrm{CPB}$ at Tokyo Women's Medical University Hospital from March 2009 to September 2009. Our policies call for administering PDE-3 inhibitors in patients with congestive heart failure or pulmonary hypertension preoperatively and in patients regarded to be at high risk of LOS post-operatively. We selected these 13 patients at random from candidates for PDE-3 inhibitor administration by having the attending anesthesiologist (a study member but not a study coordinator) draw a hidden card for each patient. These cards were either blank or bore a red mark for olprinone infusion. If a blank card was drawn, the patient was given milrinone. The group of 13 subjects administered olprinone included 7 candidates for biventricular repair and 6 
candidates for Fontan-type operation.

\subsection{Olprinone Infusion}

We initiated olprinone infusion at $0.3 \mu \mathrm{g} / \mathrm{kg} / \mathrm{min}$ with no loading dose immediately after the release of aortic cross-clamping. In cases not involving aortic crossclamping, we began the infusion immediately after all surgical procedures had been completed. The study rules did not prevent the concurrent infusion of other cardiovascular active drugs (i.e., inotropes or vasodilators). The selection and infusion doses of such agents were generally left to the discretion of the attending anesthesiologists.

\subsection{Plasma Olprinone Concentrations}

We sampled blood to measure plasma olprinone concentrations at 15, 30, 60, 90, and 120 minutes (min) after the start of infusion and at the same elapsed intervals after separation from CPB. We collected $2 \mathrm{ml}$ of plasma of each sample after centrifuging whole blood samples. We then measured plasma olprinone concentrations using ultra-fast liquid chromatography (UFLC) with a spectrofluorometric detector (Shimadzu, Kyoto, Japan). We assessed excitation/emission at 335/400 $\mathrm{nm}$. The UFLC samples were prepared by extraction using ethyl acetate with milrinone as the internal standard (IS). We separated olprinone with a Shim-pack XR-ODS column (3.0 $\mathrm{mm}$ i.d. $\times 100 \mathrm{~mm}$ ) with a mobile phase consisting of 10 $\mathrm{mmol} /$ liter phosphoric acid solution and acetonitrile $(88: 12, \mathrm{v} / \mathrm{v})$ adjusted to $\mathrm{pH} 7.0$. The analysis was performed at a column temperature of $25^{\circ} \mathrm{C}$. Retention times were $8.0 \mathrm{~min}$ for olprinone and $5.5 \mathrm{~min}$ for IS. The lowest limit of quantification was $5.0 \mathrm{ng} / \mathrm{ml}$.

\section{Results}

\subsection{Demographic Data for Study Subjects}

Table 1 summarizes the demographic data for the study subjects. Data are given as median with the range in parentheses.

\subsection{Elevated Plasma Olprinone Concentrations}

We successfully measured plasma olprinone concentrations for all 13 patients. Figure 1 illustrates the changes in plasma olprinone concentrations. The effective plasma concentration $(>20 \mathrm{ng} / \mathrm{ml})$ was achieved from $30 \mathrm{~min}$ after separation from CPB (Figure 1(a)). After dividing the patients into three groups based on the time elapsed from initiation of infusion to separation from $\mathrm{CPB}$, we found concentrations elevated above the effective concentration by $60 \mathrm{~min}$ after separation from CPB, even in patients with times of less than 30 min (Figure 1(b)).
Table 1. Data on patient demographics.

\begin{tabular}{cc}
\hline & Patients administered olprinone $(\mathrm{n}=13)$ \\
\hline Age $(\mathrm{mo})$ & $21(4-48)$ \\
Gender $(\mathrm{M} / \mathrm{F})$ & $6 / 7$ \\
BW $(\mathrm{kg})$ & $12.0(4.6-15.3)$ \\
& $\begin{array}{c}\text { VSD with PH 3, CoA complex after PAB 1, } \\
\text { Diagnosis }\end{array}$ \\
ASD with PS 1, TOF 2, SRV 3, TA 2, PPA 1 \\
Operation & $\begin{array}{c}\text { VSD closure 4, TOF correction 2, ASD } \\
\text { closure with RVOTR 1, BDG 2, TCPC 4 }\end{array}$ \\
\hline
\end{tabular}

mo: months old. M: male. F: female. BW: body weight. VSD: ventricular septal defect. PH: pulmonary hypertension. CoA: coarctation of aorta. PAB: pulmonary artery banding. ASD: atrial septal defect. PS: pulmonary stenosis. TOF: tetralogy of Fallot. SRV: single right ventricle. TA: tricuspid atresia, PPA: pure pulmonary atresia. RVOTR: right ventricular outflow tract reconstruction. BDG: bidirectional Glenn anastomosis. TCPC: total cavopulmonary connection.

With respect to time after start of infusion, we achieved effective concentrations starting $60 \mathrm{~min}$ after infusion began (Figure 1(c)).

\subsection{Side Effects}

We observed no signs of hypotension or arrhythmia with suspicious causal relationship to olprinone administration, for the duration for which the patient was administered olprinone.

\section{Discussion}

To the best of our knowledge, this study is the first to demonstrate the time course of elevations in plasma olprinone concentration after the start of infusion at weaning from $\mathrm{CPB}$ in pediatric cardiac surgery cases. The study results showed that our infusion protocol achieved effective plasma concentrations quite rapidly, within 30 min after weaning from $\mathrm{CPB}$.

The infusion procedures used in other studies have varied from study to study, and several of these studies have measured and reported blood concentrations. In the adults described in the studies reported to date, a loading dose of $15 \mu \mathrm{g} / \mathrm{kg}$ followed by continuous infusion at 0.1 $\mu \mathrm{g} / \mathrm{kg} / \mathrm{min}$ achieved effective blood concentrations within 5 min after weaning from CPB [3], while continuous infusion at 0.1 to $0.2 \mu \mathrm{g} / \mathrm{kg} / \mathrm{min}$ without a loading dose took 1.5 to 2 hours $[5,6]$. A study involving children showed that a bolus injection of $50 \mu \mathrm{g} / \mathrm{kg}$ produced elevations above the effective blood concentration within 30 min after weaning from CPB [7]. Another study confirmed levels above the effective blood concentration at 120 minutes after the initiation of continuous infusion at $0.4 \mu \mathrm{g} / \mathrm{kg} / \mathrm{min}$ in ICU following pediatric cardiac surgery in all study subjects [8]. In two other studies that did not measure plasma olprinone concentrations in adult cardiac surgery cases, continuous infusion doses 


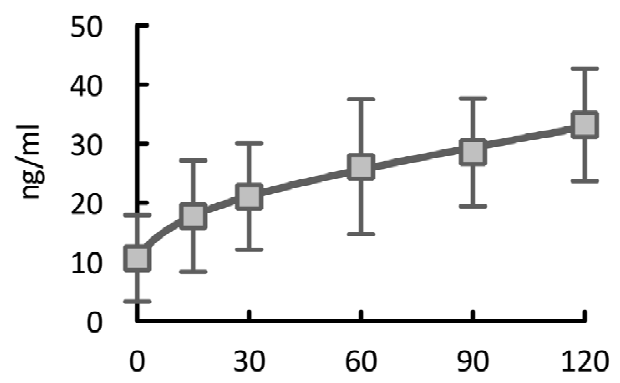

(a)

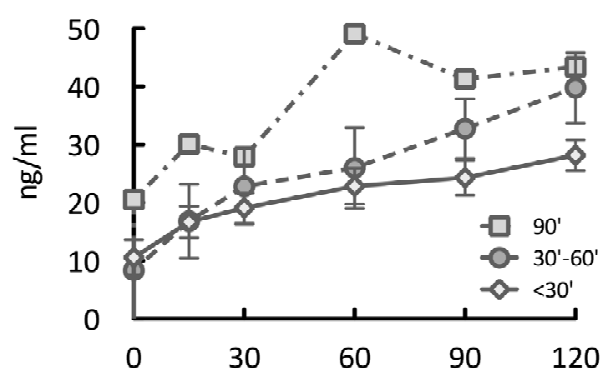

time after separation from CPB (min)

(b)

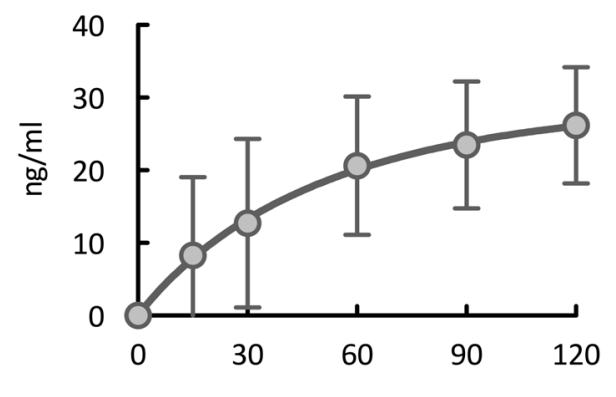

time after initiation of infusion ( $\mathrm{min}$ )

(c)

Figure 1. Time course of elevations in plasma olprinone concentration. We observed effective plasma concentrations (>20 ng/ml) starting 30 minutes (min) after separation from CPB (Figure 1(a)). Even in patients for whom the time elapsed from initiation of infusion to separation from CPB was less than $30 \mathrm{~min}$, concentrations reached or exceeded the effective concentration at $\mathbf{6 0}$ min after separation from CPB (Figure 1(b)). With respect to the time from initiation of infusion, the effective concentration was achieved from $60 \mathrm{~min}$ after initiation of the infusion (Figure 1(c)).

ranged from 0.05 to $0.3 \mu \mathrm{g} / \mathrm{kg} / \mathrm{min}$, whether or not a loading dose was administered [2,9]. Both studies confirmed that olprinone infusion at 0.1 or $0.3 \mu \mathrm{g} / \mathrm{kg} / \mathrm{min}$ achieved clinical hemodynamic stability: namely, significant tapering of concomitantly administered catecholamines [9] or significant increase in the cardiac index with reduced systemic vascular resistance [2]. Although a loading dose may assure effective blood concentrations following discontinuation of CPB, it also poses certain potential disadvantages, including the risk of BP depletion requiring noradrenaline administration [3]. We did not seek to achieve effective blood concentrations of olprinone at or before the discontinuation of $\mathrm{CPB}$; rather, our goal was simply to achieve the effective concentration shortly after separation from CPB, generally within $60 \mathrm{~min}$, thereby minimizing the risk of unwanted side effects. Given these goals, we eschewed a loading dose and high-dose infusions exceeding $0.3 \mu \mathrm{g} / \mathrm{kg} / \mathrm{min}$. Ultimately, our infusion procedure-continuous infusion at $0.3 \mu \mathrm{g} / \mathrm{kg} / \mathrm{min}$ without a loading dose after aortic clamp release or completion of the surgical procedure-achieved effective blood concentrations within just $30 \mathrm{~min}$ after weaning from CPB. Our data strongly supports the notion that the infusion procedure applied in this study is reasonable and adequate for rapidly achieving effective blood concentrations.

\section{Limitations}

Our study is subject to several limitations. First, to our knowledge, no study has been performed to date to determine the effective concentration of olprinone in children. Two earlier studies describing blood concentrations of olprinone in children have simply extrapolated an effective concentration from those for adults. We applied the same procedure in our study. Second, the sample size was small, and the study subjects varied significantly in several potentially important parameters, including age, diagnosis, pathophysiology, and severity of illness. Moreover, the extent and duration of various surgical insults, including time required for the surgical procedure, $\mathrm{CPB}$ time, and aortic cross-clamping time, differed from case to case. Some of these factors may affect the pharmacokinetics of olprinone. In the case of milrinone, Bailey et al. report that whereas $\mathrm{CPB}$ did not significantly alter the pharmacokinetics of milrinone in adults [10], certain pharmacokinetic parameters - compartment volume and clearance in the third compartment in particular-exhibited wide inter-individual variations in children aged 3 to 22 months undergoing cardiac surgery [11]. Ramamoorthy et al. have shown that milrinone does not bind to CPB circuits and that infants $(<1$ year) had small clearance compared to children ( $>1$ year), despite similar distribution volumes [12]. Ideally, a study characterized by a small sample size would control for such factors. Further studies of pharmacokinetics and the effects on circulation and oxygen delivery are needed to establish optimal olprinone infusion protocols.

In summary, the results of our study indicate our olprinone infusion procedure - continuous infusion at 0.3 $\mu \mathrm{g} / \mathrm{kg} / \mathrm{min}$ - rapidly and reliably achieved effective plasma concentrations.

\section{Acknowledgements}

We wish to thank Professor Kazuyuki Ueno at the Niigata University of Pharmacy and Applied Life Sciences, 
Faculty of Pharmacy, for assistance with measurements of olprinone plasma concentrations.

\section{REFERENCES}

[1] T. M. Hoffman, G. Wernovsky, A. M. Atz, T. J. Kulik, N. D. P. Nelson, A. C. Chang, et al., "Efficacy and Safety of Milrinone in Preventing Low Cardiac Output Syndrome in Infants and Children after Corrective Surgery for Congenital Heart Disease," Circulation, Vol. 107, No. 7, 2003, pp. 996-1002. doi:10.1161/01.CIR.0000051365.81920.28

[2] Y. Orime, M. Shiono, H. Hata, S. Yagi, S. Tsukamoto, S. Kimura, et al., "Post-Operative Effects of Olprinone after Coronary Artery Bypass Grafting," Annals of Thoracic and Cardiovascular Surgery, Vol. 4, No. 6, 1998, pp. 340346.

[3] T. Arai, Y. Takano, S. Muzukami and I. Sato, "The Effect of Olprinone Administered after Cardiopulmonary Bypass during Open Heart Surgery, Evaluated by Its Plasma Concentrations and Hemodynamic Changes (in Japanese with English Abstract)," Masui (The Japanese Journal of Anesthesiology), Vol. 48, No. 10, 1999, pp. 1083-1090.

[4] T. Arai, "Effects of Olprinone Hydrochroride after Coronary Artery Bypass Grafting (in Japanese with English Abstract)," Masui (The Japanese Journal of Anesthesiology), Vol. 53, No. 2, 2004, pp. 122-130.

[5] M. Unoshima, H. Iwasaka, S. Hattori, T. Kitano and T. Noguchi, "Effects of Olprinone Hydrochloride on Hemodynamics and Respiratory Oxygenation in Patients after Cardiac Surgery (in Japanese with English Abstract)," Masui (The Japanese Journal of Anesthesiology), Vol. 46, No. 8, 1997, pp. 1053-1058.

[6] T. Arai, K. Kamishima, T. Terauchi, Y. Enomoto, M. Shimazaki, H. Inoue, et al., "Effects of Olprinone Hydrochroride in Patients Undergoing Off-Pump Coronary Ar- tery Bypass Grafting (in Japanese with English Abstract)," Masui (The Japanese Journal of Anesthesiology), Vol. 55, No. 2, 2006, pp. 158-163.

[7] M. Itou, H. Okamoto, J. Suwa, T. Asou and S. Hoka, “Application of a PDE-3 Inhibitor, Olprinone, for Fast Track Pediatric Cardiac Surgery (in Japanese with English Abstract)," Masui (The Japanese Journal of Anesthesiology), Vol. 53, No. 3, 2004, pp. 258-263.

[8] T. Iwasaki, Y. Toda, K. Shimizu, K. Suzuki, T. Kanazawa and K. Morita, "Hemodynamic Effects of Phosphodiesterase-3 Inhibitors, Olprinone and Milrinone after Open Heart Surgery in Child of Congenital Heart Disease," Anesthesia \& Analgesia, Vol. 106, No. S1, 2008, pp. 1128.

[9] K. Sha, M. Iwata, M. Mori, Y. Motozu, N. Yonemoto, K. Kitaguchi, et al., "Evaluation of the Different Doses of Olprinone for Continuous Infusion after Cardiopulmonary Bypass for Coronary Artery Bypass Surgery (in Japanese with English Abstract)," Masui (The Japanese Journal of Anesthesiology), Vol. 52, No. 6, 2003, pp. 621-625.

[10] J. M. Bailey, J. H. Levy, M. Kikura, F. Szlam and C. C. Hug Jr., "Pharmacokinetics of Intravenous Milrinone in Patients Undergoing Cardiac Surgery," Anesthesiology, Vol. 81, No. 3, 1994, pp. 616-622. doi:10.1097/00000542-199409000-00014

[11] J. M. Bailey, B. E. Miller, W. Lu, S. R. Tosone, K. R. Kanter and V. K. Tam, "The Pharmacokinetics of Milrinone in Pediatric Patients after Cardiac Surgery," Anesthesiology, Vol. 90, No. 4, 1999, pp. 1012-1018. doi:10.1097/00000542-199904000-00014

[12] C. Ramamoorthy, G. D. Anderson, G. D. Williams and A. M. Lynn, "Pharmacokinetics and Side Effects of Milrinone in Infants and Children after Open Heart Surgery," Anesthesia \& Analgesia, Vol. 86, No. 2, 1998, pp. 283289. doi:10.1097/00000539-199802000-00011 